\title{
Does One-off Accidental Exposure to Organic Solvents cause the Chronic Symptoms of Poisoning?
}

\author{
Hiroshi Ujiie ${ }^{1,2 *}$, Hanae Terashima ${ }^{2}$ and Kouichi Katoh ${ }^{3}$ \\ ${ }^{1}$ Research Institute of Ujiie Neurosurgical \& Medical Clinic, Japan \\ ${ }^{2}$ Department of Neurosurgery, Kamagaya General Hospital, Japan \\ ${ }^{3}$ Department of Neurosurgery, Tokyo Rosai Hospital, Japan
}

*Corresponding author: Hiroshi Ujie, Research Institute of Ujiie Neurosurgical \& Medical Clinic,102-0094, 3-19 Kioi-cho, Chiyoda-

city, Tokyo, Japan

\section{ARTICLE INFO}

Received: 幽 September 23, 2020

Published: 㐩 October 01, 2020

Citation: Hiroshi Ujiie, Hanae Terasima, Kouichi Katoh. Does One-off Accidental Exposure to Organic Solvents cause the Chronic Symptoms of Poisoning?. Biomed J Sci \& Tech Res 30(5)-2020. BJSTR. MS.ID.005017.

\begin{abstract}
It is well known that the symptoms of acute poisoning after exposure to organic solvents decrease with time and the acute symptoms do not last for a long time. However, it is sometimes encountered that the poisoning symptoms in the acute phase might become chronic in some cases. Here we report a case with the symptom of purpura and severe headache after acute poisoning by organic solvents. The symptom is continuing even after years, and many medical treatments were failed. The mechanism of purpura and headache even in chronic stage was based on vasculitis after contamination of vessel walls by organic solvents. In this case, inflammation created in perivascular area must be origin of such purpura and hypertrophic pachymeningitis in the temporal base that inducing severe headache.
\end{abstract}

Keywords: Organic Solvent; Solvent Poisoning; Headache; Pachymeningitis

\section{Introduction}

Organic solvents are the most frequently encountered chemicals used in daily work, but due to their high volatility and fat solubility, they are easily absorbed into the body through the respiratory tract and cause toxic symptoms. Also, a certain amount is absorbed from skin contamination. Inhalation of highly concentrated organic solvents instantaneously causes acute poisoning, and long-term exposure also causes chronic symptoms [1]. It is well known that the symptoms of acute poisoning after exposure to organic solvents decrease with time and the acute symptoms do not last for a long time. However, it is sometimes encountered that the poisoning symptoms in the acute phase might become chronic in some cases. Here, we report a case in which headache and skin symptoms persisted after a long time after one occasional history of acute poisoning of organic solvents.

Case 1

Patient: 53-year-old woman.
Chief Complaint: Chronic severe headache and purpuric ulcer dermatitis after acute organic solvent poisoning.

\section{Family history: No history of craniotomy.}

Present Illness: On October 9, 2014, she parked the vehicle in the underground parking where another vehicle was waxed next parking place. It was accidentally happened that she inhaled dense organic solvent volatilized from the wax. Immediately after inhaling the gaseous organic solvent, nausea and headache occurred, 1 hour later, dizziness, diarrhea, vomiting, headache and 3 hours later, numbness in the whole body and immobility followed. These symptoms are kind of severe acute poisoning. Gastrointestinal symptoms improved within a month, but severe headache continued thereafter. In addition, a red rash appeared on the exposed forearm, back of the hands, face, lower extremities and neck. Then headache and such dermatitis persisted despite a single exposure to organic solvents and bothered her life every day. She visited the Neurosurgical department of Tokyo Rosai 
Hospital about two years later. The headache was throbbing type and it started characteristically 3 hours later when lying on the bed, she had to wake up to take a sitting position because only sitting position relieved headache. Daily life was very limited due to lack of sleep and persistent headaches.

Before coming to our department, she visited on a poisoning outpatient department, a neurology department, and several neurosurgery departments and anesthesia departments, and many drugs had already been given. However, NSAIDs, triptan, ergotamin are not effective. Also, tranquilizers and antiepileptic drugs were prescribed, but rather, nausea, weakness, and worsening of headache occurred, and she could not continue taking the drug. In our department, hyperosmotic diuretics were found to be effective for headache. A dermatologist diagnosed ulcerative dermatitis that remained in both lower limbs as chronic purpura. Other dermatitis was cured.

A. Blood Examination: Normal blood glucose level, neither liver nor renal dysfunction, negative ANCA.

B. Neurological Findings: There were no neurological abnormalities, no depressive symptoms, and WAIS has shown a high score.

C. Neurophysiological and Radiological Findings: EEG did not show spike waves suggesting seizures.

D. Diagnostic Imaging: MRI was done in several hospitals, but T1 image, T2 image, Flair, and MRA showed no abnormalities. However, coronal view of contrast-enhanced MRI that was taken first in Tokyo Rosai hospital showed thickening of the dura in the middle skull base with positive contrast enhancement. This finding was considered as hypertrophic pachymeningitis (Figure 1).

E. Ophthalmological Findings: Normal intraocular pressure, orthonormal position, and glasses were appropriate.

F. Dermatology: purpura in both lower limb (Figure 2).
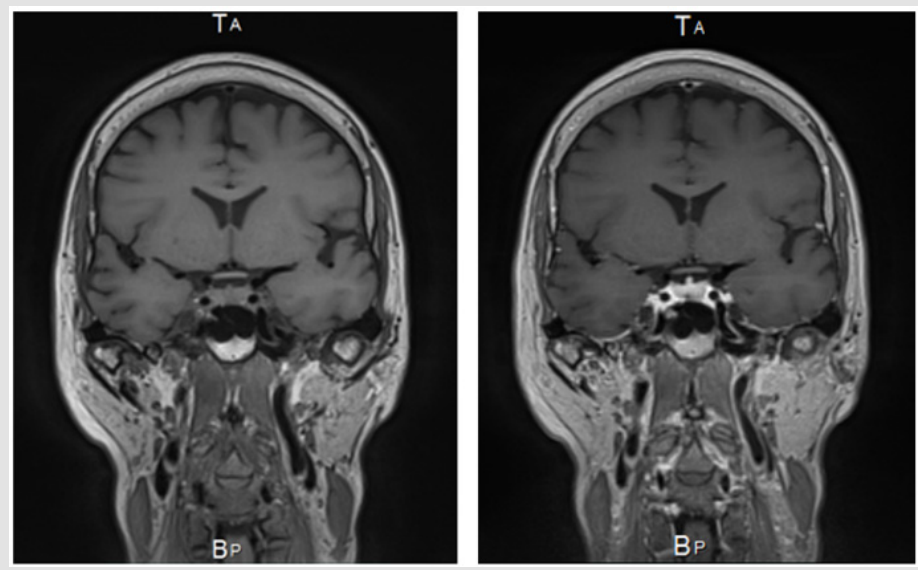

Figure 1: MRI scans with and without Gd. Lt side, coronal image T1, Rt side, coronal image of same position with Gd enhancement, linear dural thickening along bilateral middle fossa dura pointed out.

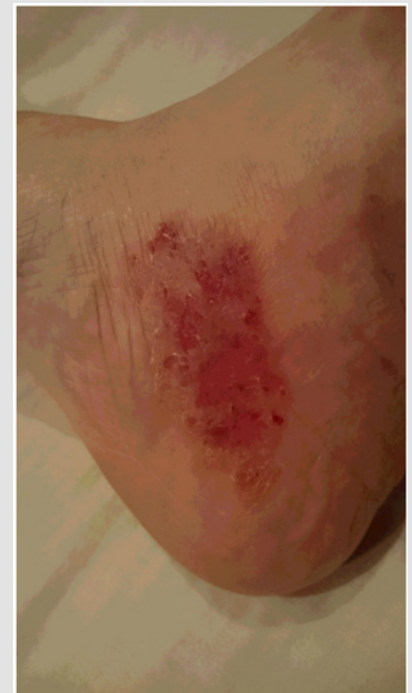

Figure 2: Purpura noted on the left lower limb. 


\section{Discussion}

\section{Organic Solvent Poisoning}

Symptoms of acute intoxication of organic solvents are recovered after the organic solvents being metabolized and excreted in the urine. Further, when it remains in the body, the organic solvent is first metabolized intracellularly by cytochrome P450 and finally it is conjugated by glucuronic acid and glutathione, becomes water-soluble and is excreted through the kidney. Generally, it is said that the chronic symptom of the organic solvent occurs when continuously exposed to a low concentration and the case where the acute symptom has remained at the chronic phase never reported.

\section{Properties of the Organic Solvent used this Time}

An automotive polish wax was Carb right including 1,2,4 trimethylbenzene $<1$ WT\%, 1,3,5 trimethylbenzene $<1$ WT\%, ethylbenzene 0.7 WT\%, xylene 1.5 WT\%, naphthalene $<1$ WT\%, nonane 0-10 WT\%, mineral spirits 60-70 WT\%, silicone oil 0-10 WT\%, benzene derivatives $(1,2,4 \& 1,3,5$ trimethylbenzene, ethylbenzene, xylene, naphthalene) about 5 WT\%. The main organic solvent dissolved in silicone oil is heavier than air. The organic solvents (5WT\% benzene derivatives) may sink below and exist in a high concentration locally around it is used (Figure 3 ). Since the air moves on a certain route from the wind outlet of ventilator to ventilating hole, so the components of the volatilized organic solvent do not spread evenly throughout the room. There must be a high concentration area in the parking room. The patient must inhale the high-concentration organic solvent vapor sitting in the driver seat when open the car door. One or two breath of dense organic solvent must cause acute poisoning.

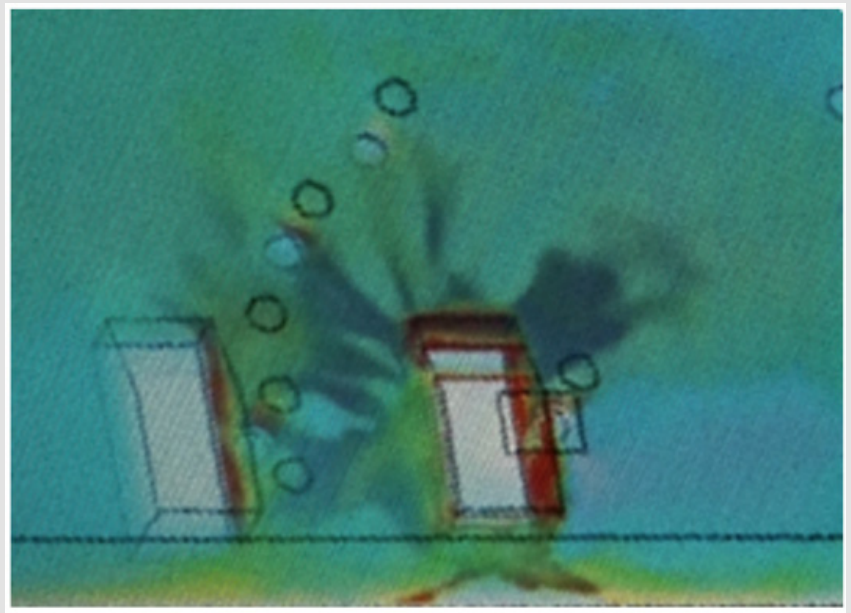

Figure 3: Computer simulation study of air flow in the parking lot. Lt hand, patient's car and rt hand side man waxed on the roof. Patient just opened rt side door and breathed several times of high-level organic solvents stagnant around and under the patient car.

\section{Causal Relationship between Severe Headache and} Enhanced Hypertrophic Dura Matter

The headache in this case is exacerbated by reclining. It is speculated that this was caused by a decrease in hydrostatic pressure due to recumbency, resulting in a decrease in venous drainage of cerebrospinal fluid and an increase in intracranial pressure. Coronal image demonstrated linear dural thickening and enhancement along the middle cranial fossa shown in Figure 1. The middle cranial fossa dura matter is fed by meningeal arteries that are rich in sensory nerves from the trigeminal nerve. Unmyelinated nerve fibers derived from the trigeminal ganglion are distributed to the dural arteries and serve to transmit the noxious pain $[2,3]$.

\section{In Conclusion}

Organic solvents are easily absorbed and caused acute poisoning, however if it was single exposure, it was metabolized and evacuated quickly from each organ. But invasion of organic solvent into the vascular wall caused inflammation of the vascular wall and perivascular soft tissues. Poor microcirculation of dura matter and skin of the lower limb failed to cure the inflammation. This must be the reason of chronic vasculitis after single exposure of organic solvents. It is well known that skin vasculitis is common symptom after organic solvents contamination [4]. Such vasculitis occurred to the everywhere in the body. The patient's vasculitis remained only in dura matter and lower limb where microcirculation is not enough to evacuate inflammation substances. This case taught us single exposure of organic solvents caused vasculitis in the skin and severe headache, if patient's headache exacerbated by recumbency, Gd-enhanced MRI is necessary to prove the inflammation of dura matter.

\section{References}

1. Winder C, Turner PJ (1992) Solvent exposure and related work practices amongst apprentice spray painters in automotive repair workshops. Ann Occup Hyg 36(4): 385-394. 
2. Asger MS, Hansen AE, Kapijimpanga T, van der Geest RJ, van der Koning P, et al. (2010) Dilatation by CGRP of middle meningeal artery and reversa by sumatriptan in normal volunteers. Neurology 75(17): 1520-1526.

3. Amin FM, Asghar MS, Guo S, Hougaard A, Hansen AE, et al. (2012) Headache and prolonged dilatation of the middle meningeal artery by PACAP38 in healthy volunteers. Cephalalgia 32(2): 140-149.

\section{ISSN: 2574-1241}

DOI: 10.26717/BJSTR.2020.30.005017

Hiroshi Ujiie. Biomed J Sci \& Tech Res

(C) This work is licensed under Creative

Submission Link: https://biomedres.us/submit-manuscript.php
4. Maki S, Nawata H, Ogawa Y (2011) Analysis of organic solvent poisoning occurring in Japan from 1995 to 2006. San Ei Shi 53(3): 87-100.

\begin{tabular}{|ll}
\hline & Assets of Publishing with us \\
\hline RESEARCHES & - Global archiving of articles \\
\hline
\end{tabular}

